\title{
METHODS FOR ASSESSING THE TECHNICAL CONDITION OF GAS TURBINE ENGINE (GTE) BASED ON STRENGTH CRITERIA
}

\author{
Olala M. Olali ${ }^{1}$, Tolumoye J. Tuaweri ${ }^{2}$, Nataliya. V. Olali ${ }^{3}$ \\ ${ }^{I}$ Department of Mechanical/Marine Engineering, Niger Delta University, Wilberforce Island, Bayelsa State, Nigeria \\ ${ }^{2}$ Department of Mechanical/Marine Engineering, Niger Delta University, Wilberforce Island, Bayelsa State, Nigeria \\ ${ }^{3}$ Department of Mathematics/Computer Science, Niger Delta University, Wilberforce Island, Bayelsa State, Nigeria
}

\begin{abstract}
This paper deals with a method of diagnostics and focuses on the estimation of the depletion or exhaustion of service life of components of gas turbine engine. During operation of the gas turbine engine the critical components of the engine; blades, discs, shafts, wheels, gears, gearboxes and drive assemblies experience the action of centrifugal and gas forces that bring about tension, bending and turning moments. They also experience thermal loads of high intensity that has negative effect on the strength of the materials of the components. The strength of these components defines the reliability of the engine. The forces and thermal loads acting on the critical elements lead to the depletion of the service life of these elements. Hence the estimation of the remaining service life of gas turbine engine components.
\end{abstract}

Keywords: Blades, critical components, damage, depletion, gas turbine engine, failure, service life, typical flight

\section{INTRODUCTION}

The need to assess the technical condition of engines on the strength criteria is dictated by the increase in their service life and significant differences of operating conditions of each individual turbine engine. The need to improve the relevance of these techniques is continuously increasing, as the engines with higher turbine inlet temperature, small variations in temperature and mechanical loads lead to intensive service life exhaustion of critical structural elements. The critical elements include rotor blades, wheels of the turbocompressor, bearings of rotor support, gears, gearboxes and drive assemblies and shafts. Fan blades, compressor and turbine disks can be considered as controllable units. One of the most loaded parts of the engine are the blades of the turbo compressors, experiencing the action of centrifugal and gas forces. Centrifugal forces cause tension, bending and torsion of the blades. The most significant of these are tensile stresses. Discs of turbo compressors are some of the most critical elements that define gas turbine engine reliability. Destruction of discs are extremely dangerous, as flying debris (fragments), having large mass and speed at the time of the destruction, can cause severe damage to the secondary structural elements of the engine and the aircraft, so the issues of strength of discs are very important. Individual elements of the rotors and casings experience various forces and thermal exposure of high intensity that bring about negative influence on the strength of materials. Some of the most loaded elements are shafts, spline, dowels and strain pin connections (coupling) of the rotor and casings. Turbine shafts absorb all types of loads acting on the rotor. These loads can be divided into internal (produced by rotor elements during engine operation) and external, which arise during the evolution of the aircraft in flight. Failure of these elements have a direct impact on flight safety [1-4].

\section{ANALYSIS OF RESEARCH WORKS AND PUBLICATIONS}

Addressing the issue of accounting for the depletion of service life of Gas Turbine Engine (GTE) on critical structural elements began with the development of methods and means of recording the operating (running) time of engines in operation. Since the engine operation conditions in the various airlines differ significantly because of the geographical location and duration of flights, then recording the operating time alone is not an objective measure. In this case, the different forces and thermal loads acting on the elements of the design of the engine when it is operating under different conditions is not taken into account.

One method of assessing its service life, taking into account the loading of structural elements and operating conditions of GTE was developed by V.I.Yampolsky with regards to a working turbine blade [5]. According to this method, the residual service life is defined by the formula

$$
\mathrm{t}_{\mathrm{res}}=\mathrm{t}_{\mathrm{ss}}-\int_{0}^{t}\left(\frac{t_{s}}{t}\right) \mathrm{dt}
$$

where $t_{s s}$ - service life while the engine is at steady-state conditions for typical conditions;

$\mathrm{t}_{\mathrm{s}}-$ service life

$\mathrm{t}$ - total operating time of the GTE at steady-state conditions.

Service life of the rotor blade is considered expended when the likelihood that the current stresses acting in it have exceeded the limit, have reached an unacceptable value. With a normal distribution of random variables of stresses acting in the blade, the likelihood of its destruction is given by: 


$$
\mathrm{p}=\frac{1}{2}-\mathrm{F}\left[\left(\mathrm{t}_{\lim }-\mathrm{T}\right) / \mathrm{d}\right]
$$

where $\mathrm{T}$ - the expectation of effective stress $t_{\text {lim }}$ - the expectation of stress limit

d- the standard deviation of the difference between the random variables $\left(\mathrm{t}_{\mathrm{lim}}-\mathrm{T}\right)$;

$$
\mathrm{F}\left[\left(\mathrm{t}_{\lim }-\mathrm{T}\right) / \mathrm{d}\right]-\text { Laplace's tabulated function }
$$

With known dependencies of $\mathrm{T}, \mathrm{t}_{\lim }$ and $\mathrm{d}$ on the rotor speed of the engine, the gas temperature at the turbine inlet (TIT), the rate of depletion of the service life and the probability of failure of the blade can be determined. The service life is considered exhausted if the value $\alpha=\left(\mathrm{t}_{\lim }-\mathrm{T}\right) / \mathrm{d}$ reaches five [5-6]. Damage to structural elements are conventionally divided into visible and invisible. Visible damage are revealed by Non Destructive Testing (NDT) methods or indirectly by the change in the level of vibration of rotor supports [5-6].

Invisible damage accumulated since the beginning of operation in micro- structural elements are evaluated by calculation methods. Computational methods allow us to establish a connection between the parameters characterizing the conditions in which the structural elements of the engine operate and the intensity of depletion of their durability. These methods are based on a comparison of the damage accumulated during the implementation of the $\mathrm{j}$-th flight and those accumulated for a typical flight. Accumulated amount of damage per flight for known loading parameters for a particular part is evaluated using the following relation [5-7] :

$$
\mathrm{d}_{\mathrm{j}}=\sum_{i=1}^{n_{j}} \frac{t_{i}}{\tau_{i}^{i}}
$$

where $n_{j}$ - the number of working modes of GTE for $j$-th flight

$t_{i}$ - the duration of the $i$-th mode

$\tau_{i}$ - durability of the parts in which parameters characterize the $i$-th mode of the engine

For a typical flight the amount of damage $\mathrm{d}_{\mathrm{TF}}$ can be determined by a formula similar to (3)

$$
\mathrm{d}_{\mathrm{TF}}=\sum_{i-1}^{n_{\mathrm{tg}}} \frac{t_{\mathrm{mi}}}{\tau_{F i}}
$$

Blades and discs are subjected to complex loading and to evaluate their loading value of integrated stresses that take into account the effect of static, thermocyclic and vibration stresses on their durability are used. In the case of complex loading on structural materials of blades and turbine discs [8-9], durability characteristics is described by the equation

$$
\lg \tau=\mathrm{a}+\mathrm{b} \sigma_{\mathrm{k}}
$$

where $\mathrm{a}$ and $\mathrm{b}$ - coefficients that depend on the magnitude of the temperature of the parts in the section with maximum loads $\mathrm{T}_{\mathrm{k}} ; \sigma_{\mathrm{k}}-$ complex load
Within the limits of analysis the temperature changes within the blades and discs of the turbo compressor when the engine operating mode changes; the relationship of the coefficients $\mathrm{a}$ and $\mathrm{b}$ with respect to temperature is close to linear. That is the following equations are valid;

$$
\mathrm{a}=\alpha+\beta \mathrm{T}_{\mathrm{C}}
$$

$$
\mathrm{b}=\gamma+\lambda \mathrm{T}_{\mathrm{C}}
$$

where $\alpha, \beta, \gamma, \lambda$ - constants for the material of construction of the part under consideration [10-11].

To assess the damage of component parts of the turbo compressor, equations (3) and (4) are used, transformed and taking into account equations (5) and (6) we have

$$
\begin{array}{r}
\left(\mathrm{d}_{\mathrm{j}}\right)_{\mathrm{TC}}=\sum_{i=1}^{n j} \frac{t_{\mathrm{i}}}{\alpha+\beta T_{\mathrm{Ci}}+\left(\gamma+\lambda T_{C \mathrm{i}}\right) \sigma_{\mathrm{Ci}}} ; \\
\left(\mathrm{d}_{\mathrm{TF}}\right)_{\mathrm{TC}}=\sum_{\mathrm{i}=1}^{n_{T F}} \frac{t_{\mathrm{i}}}{\alpha+\beta\left(T_{\mathrm{C}}\right)_{T F i}+\left[\gamma+\lambda\left(T_{C}\right)_{T F i}\right] \sigma_{C \mathrm{i}}}
\end{array}
$$

With information about the number of flights $\mathrm{Z}$ of the engine under investigation, the accumulated damage for real $\mathrm{d}_{\mathrm{R}}$ and model(typical) flight $\mathrm{d}_{\mathrm{TF}}$ is determined by summation and

$$
\mathrm{d}_{\mathrm{R}}=\sum_{j=1}^{z} d_{j} \text { and } \mathrm{d}_{\mathrm{TF}}=\sum_{j=1}^{z}\left(d_{T F}\right)_{j}
$$

The quantities $d_{R}$ and $d_{T F}$ are considered as diagnostic parameters but their residuals $\left(\Delta \mathrm{Q}=\mathrm{d}_{\mathrm{R}}-\mathrm{d}_{\mathrm{TF}}\right)$ as diagnostic signs.

For serviceable engine the following condition must be satisfied

$$
\Delta \mathrm{Q}_{\mathrm{T}} \leq \mathrm{Q}_{\lim }
$$

where $\mathrm{Q}_{\mathrm{lim}}$ - is the limiting value of the residual.

The value of $\mathrm{Q}_{\lim }$ is determined for the most unfavorable loading conditions when the engine is considered to be in a proper and workable condition. If condition (9) is not satisfied, the GTE is subjected to careful quality control. For example, if the damage to the turbine blades increases dramatically, then it needs to be examined using borescope. To estimate the intensity of the process of exhaustion of the service life, the value of the equivalent operating time is used which is

$$
\tau_{\text {equ }}=\frac{t_{T F}}{d_{T F}} \mathrm{~d}_{\mathrm{j}}
$$

The remaining service life $t_{\text {rem }}$ in this case is determined by the formula

$$
\mathrm{t}_{\mathrm{rem}}=\mathrm{t}_{\mathrm{R}}-\sum_{j=1}^{z} \tau_{e q u j}
$$

where $t_{R}-$ service life of the Gas Turbine Engine GTE. 


\section{CONCLUSION}

A method of estimating the depletion or exhaustion of service life of components of gas turbine engine is offered. The use of equivalent operating time makes the method easier than V.I. Yampolsky's method. The offered method of diagnostics is suitable for airlines whose aircraft or helicopters are equipped with gas turbine engines. The method is also suitable for turbo- mach inery installations used for transportation of fluids or production of electricity, etc.

\section{REFERENCES}

[1] Kruckenberg H.D. Evaluation of American airlines B747 prototype aids engine monitoring program. "AIAA Paper”, 1971, № 648, 10 pp., ill

[2] Neese W.A. Use of expander AIDS in engine health monitoring on the GF6-80 engine for the A310 airbus. “SAE. Techn. Pap. Ser.”, 1982. №841505. P.1-13

[3] Panin V., Voznyuk A., Sun G., Olali M. Gas turbine engine diagnosing complex method development on the basis of multiple factors analysis. ISSN 18131166. Proceedings of the NAU. 2006. № 3. P.49-52.

[4] Boguslayev A.V Kagan AY, Popov V.V. The impact of damages on the endurance of rotor blades of the compressor GTE // Aviation-space technics i technologies. - Kharkiv, 2002. - Vol.32. - P.115-118.

[5] Lozitsky L.P, Janko A.K, Lapshov V.F. Evaluation of the technical condition of gas turbine engines. - M .: Transport, 1982. - $160 \mathrm{p}$.

[6] Lapshov V.F. Management Basics on technical condition of aircraft gas turbine engines in operation. - Kiev: KIIGA, 1989. - 108 pages.

[7] Kulik N.S.Parametric methods for assessing the technical state of gas turbine engines. - Kiev: KIIGA, 1993.-139 pages.

[8] Klyuev V.V. Non-destructive testing. Mechanical Engineering, 2006. - 829 pages

[9] Simbirsky D. F, Oleynik A. V, Filyaev V. A and others. Programable system for operational monitoring of the depletion of service life of the main parts of aviation engine D-18T // Aerospace equipment and technology. -X .: NAKU "HAI" .2002.iss. 7/15. - P.145-150.

[10] Janko A.K. Determination of residual life of GTE based on the analysis of changes in the strength margin of the most loaded elements / / Industrial Heat. -Kiev Sciences. Dumka, 1980, № 2. - P 231240.

[11] Friedman Y.B. Mechanical properties of metals. M.: Mechanical Engineering, 1972. 